\title{
Evolución y estado actual del e-learning en la Formación Profesional española
}

\section{The development and current situation of e-learning in Spanish Vocational Training}

\author{
Cristian Jorge García Marcos \\ Universidad de Sevilla (España) \\ Julio Cabero Almenara \\ Universidad de Sevilla (España)
}

\section{Resumen}

El artículo está basado en un estudio cuyo objetivo es el análisis de los modelos organizativos a nivel institucional, las herramientas tecnológicas y los recursos didácticos que caracterizan las enseñanzas de formación profesional a distancia en todo el territorio español. Para la recogida de datos se emplearon técnicas cuantitativas y cualitativas. Los resultados más relevantes del estudio fueron: constatación de un aumento continuo en el número de estudiantes matriculados en los estudios a distancia de formación profesional, uso por parte de algunas Comunidades Autónomas de materiales didácticos creados específicamente para dicha modalidad de enseñanza y que son distribuidos en formato abierto, hegemonía absoluta en el empleo de Moodle como entorno virtual de aprendizaje y de sus herramientas de comunicación asíncronas y síncronas, diferencia entre Comunidades Autónomas con respecto a la ratio de estudiantes por módulo y con respecto a la carga horaria docente dedicada a la acción tutorial, e implantación a nivel nacional de la mayoría de la oferta formativa de formación profesional. Además, se ha creado un mapa interactivo que recoge parte de la información recopilada en el estudio. A partir de los resultados encontrados, se constata que existen ciertos aspectos que difieren significativamente entre Comunidades Autónomas y que podrían subsanarse, por un lado, a través de una mayor comunicación y coordinación entre ellas y, por otro, mediante el intercambio de experiencias.

Palabras clave: educación; aprendizaje en línea; formación profesional.

\begin{abstract}
This paper is based on a study aimed to analyze organizational models at institutional level, technological tools and educational resources that distinguish online vocational studies throughout the Spanish territory. Quantitative and qualitative techniques were used for data collection. The most relevant results of the study were: there is an increase in the number of students enrolled in vocational training distance learning studies, materials specifically created and distributed in an open format for distance learning are used in some regions, absolute hegemony of Moodle as a virtual learning environment with its synchronous and asynchronous
\end{abstract}


communication tools, existence of a gap between regions regarding the ratio of students per module and the hours devoted to tutorials, distance learning national implementation for the most vocational training studies, and creation of an interactive map specifically in order to show information collected in the study. Considering this situation, there are certain aspects which differ significantly among regions and which could be improved in two ways: firstly, throughout better communication and coordination, and secondly, by means of exchanging experiences between regions.

Keywords: education; electronic learning; vocational education.

No cabe la menor duda que la formación virtual, el e-learning, la teleformación, o como queramos denominarla, se está convirtiendo en una de las estrategias de formación más utilizada en los últimos tiempos, tanto en la educación formal como en los contextos profesionales y empresariales. Y posiblemente su utilización evolucione de forma constante en los próximos años. Como se indica en un post del blog "Origin Learning" denominado "10 Facts about e-learning"1 se espera que en el año 2016 el mercado de e-learning de Asia ascienda a 11.5 billones de dólares, y que se incremente en una tasa promedio del 23\% hasta el año 2017.

En el estudio que se presenta se analiza el estado actual y la evolución que ha tenido el e-learning en las enseñanzas de Formación Profesional a distancia (FPaD) en España en general, y de manera particular en cada una de las Comunidades Autónomas. En concreto, se han analizado los modelos organizativos a nivel institucional, las herramientas tecnológicas y los recursos didácticos que caracterizan los estudios a distancia de la formación profesional española bajo esta modalidad de formación.

Y para ello es importante señalar desde el comienzo y de acuerdo con las propuestas de diferentes autores (Ellis y Goodyear, 2010; Brazuelo y Gallego, 2011; Thorne, 2012; Aguaded y Cabero, 2013; Castaño y Cabero, 2013; Cabero, 2015; Salinas, Darder y De Benito, 2015; Sevillano y Vázquez, 2015; Torres, Infante y Torres, 2015), que vamos a entender por esta modalidad de formación la apoyada en la presentación de contenidos a través de Internet y que utiliza diferentes herramientas de comunicación sincrónicas y asincrónicas para establecer la interacción entre docente y discente. De todas formas no debemos olvidarnos de las matizaciones que realiza García Aretio (2014) cuando nos especifica que la misma debe tener cuatro características básicas: separación física docente y discente, el estudio independiente por parte del alumno, comunicación e interacción entre los participantes en el acto instruccional mediante diferentes herramientas de comunicación sincrónicas y asincrónicas, y la existencia de un soporte institucional.

De manera específica, aunque existen ciertas enseñanzas de e-learning en la FPaD española que han sido diseñadas para ser implementadas de manera completamente virtual, gran parte del e-learning en este nivel se basa en un modelo de aprendizaje mezclado o blended learning (Marsh, McFadden y Price, 2003; Bartolomé, 2004; 
Dukes, Waring y Koorland, 2006), en la que la formación virtual se combina parcialmente con la formación presencial.

\section{ANTECEDENTES EN LA LITERATURA}

Como afirman Duarty Lupiáñez (2005) “[...]la realidad del e-learning es compleja. Las tecnologías de la información y de la comunicación (TIC), y especialmente Internet, han abierto nuevas perspectivas en el horizonte de la educación en el siglo XXI". Son varios los autores que han estudiado esta complejidad a través de diversos modelos que se componen de un número diferente de dimensiones. Así, Salinas (2004) ha elaborado un modelo basado en tres dimensiones; Sangrà (2008) emplea cinco dimensiones; García Aretio (2014) indica tres dimensiones en interacción; y Khan (2001 y 2015) propone uno formado por ocho dimensiones. El común denominador de todos estos modelos son las tres dimensiones básicas que se presentan en el cuadro 1, y en las cuales se basa el presente estudio:

- Dimensión pedagógica. En ella aparecen elementos que tratan sobre la planificación y el desarrollo del proceso de enseñanza-aprendizaje apoyado en el uso de las TIC.

- Dimensión tecnológica. Contiene elementos relacionados con la infraestructura física y lógica que permiten el despliegue adecuado del proceso de enseñanzaaprendizaje a distancia.

- Dimensión organizativa. Donde se incluyen elementos acerca de los aspectos institucionales que afectan a toda la comunidad educativa.

Dentro de estas dimensiones, se han establecido una amplia variedad de elementos críticos del e-learning. De entre todos estos elementos, en el presente estudio únicamente se han tratado aquellos que son susceptibles de ser analizados a nivel general, es decir, aquellos que afectan por igual a toda la comunidad educativa, como por ejemplo el número de horas que un docente tiene asignadas para la dedicación a la tutoría por cada módulo formativo. Los elementos que se desarrollan en un nivel mayor de concreción, como puede ser el método de evaluación aplicado por un docente para conocer el nivel de aprendizaje de un estudiante, quedan fuera del alcance de los objetivos de la investigación. 
Cuadro 1. Dimensiones del e-learning y elementos analizados para cada dimensión

\begin{tabular}{|c|c|c|}
\hline DIMENSIÓN & ELEMENTO & ESTUDIOS DE REFERENCIA \\
\hline \multirow{3}{*}{ Pedagógica } & Materiales didácticos & $\begin{array}{l}\text { Salinas (2005), Cabero (2006), Area y Adell } \\
\text { (2009), García Aretio (2014), Khan (2015) }\end{array}$ \\
\hline & Comunicación e interacción & $\begin{array}{l}\text { Salinas (2005), Cabero (2006), Area y Adell } \\
\text { (2009), García Aretio (2014), Khan (2015) }\end{array}$ \\
\hline & $\begin{array}{l}\text { Tamaño de clase y carga de } \\
\text { trabajo (tutoría) }\end{array}$ & $\begin{array}{l}\text { Cabero (2006), Area \& Adell (2009) Khan } \\
\text { (2015) }\end{array}$ \\
\hline \multirow[t]{2}{*}{ Tecnológica } & $\begin{array}{l}\text { Entorno virtual de } \\
\text { aprendizaje }\end{array}$ & $\begin{array}{l}\text { Salinas (2005), García Aretio (2014), Khan } \\
\text { (2015) }\end{array}$ \\
\hline & Recursos tecnológicos & García Aretio (2014), Khan (2015) \\
\hline \multirow[b]{2}{*}{ Organizativa } & $\begin{array}{l}\text { Marco institucional } \\
\text { (modelo de organización) }\end{array}$ & Salinas (2005), Cabero (2006) \\
\hline & $\begin{array}{l}\text { Estrategia de } \\
\text { implementación } \\
\text { (oferta formativa) }\end{array}$ & Salinas (2005), Cabero (2006) \\
\hline
\end{tabular}

Para finalizar, simplemente señalar dos aspectos: 1) que son pocos los estudios que se han realizado en nuestro contexto para el análisis de las enseñanzas bajo la modalidad e-learning en la formación profesional reglada, y 2) que a pesar de lo limitado de los estudios en e-learning, esta modalidad está apareciendo como una estrategia formativa de verdadera importancia para la capacitación de las personas en los ámbitos de la formación profesional y de la formación profesional para el empleo (Núñez, 2011; Fundación Apel, 2012; Díaz, Álvarez y Rodríguez, 2013; Vega, 2013), alcanzando resultados significativos en este colectivo, bien sea por la adquisición de nuevas competencias profesionales, bien por la necesidad de ampliación de la formación de las personas que están ocupadas, o bien por el retorno a acciones formativas suspendidas en su momento por diferentes motivos.

\section{OBJETIVOS}

El presente estudio tiene el siguiente objetivo general:

"Analizar los modelos organizativos a nivel institucional, junto con las herramientas tecnológicas y los recursos didácticos que caracterizan a los estudios de formación profesional a distancia en todo el territorio español”. 
Este objetivo general se concreta en los siguientes objetivos específicos:

- Identificar los centros educativos públicos de todo el territorio español donde se imparten enseñanzas de formación profesional a distancia y los estudios que se ofertan en cada uno de estos centros.

- Analizar ciertos elementos críticos de índole pedagógica, tecnológica y organizativa del e-learning empleados por los centros públicos que ofertan estudios a distancia de formación profesional.

- Diseñar y construir un mapa virtual interactivo que permita acceder a la información recogida en la presente investigación.

\section{METODOLOGíA}

Para el alcance de los objetivos declarados anteriormente en nuestra investigación hemos llevado a cabo una metodología de tipo mixto, apoyada por una parte en el análisis de contenido de diferentes documentos como son los sitios web oficiales de diferentes instituciones públicas que se centran en la formación profesional a distancia, y la recogida de información de diferentes expertos existentes en las Comunidades Autónomas que se dedicaban a la puesta en acción de la FPaD.

La investigación se desarrolló en tres fases, en las cuales realizamos las actividades que señalamos a continuación:

- Fase 1. Identificación de los centros educativos públicos de todo el territorio español donde se imparten enseñanzas de FPaD y los estudios que se ofertan en ellos.

- Fase 2. Análisis de elementos críticos de e-learning a nivel organizativo, pedagógico y tecnológico que se emplean por los centros públicos que ofertan estudios de FPaD.

- Fase 3. Diseño y creación de un mapa virtual que permita el acceso a la información recogida en el estudio clasificada por Comunidades Autónomas con enseñanzas de FPaD.

\section{Procedimiento utilizado para la Fase 1}

En la Fase 1 se realizó una recogida de datos con la relación de las Comunidades Autónomas que imparten enseñanzas de FPaD. Tras obtener esta información, se investigó cuáles eran los centros públicos que impartían dichas enseñanzas y los estudios que se ofertaban en cada uno de ellos. Finalmente, se identificó el "Entorno Virtual de Aprendizaje” (EVA) utilizado en cada Comunidad Autónoma. 
La recogida de información se estableció a través de dos mecanismos: análisis de las páginas web oficiales de las diversas administraciones públicas que tienen competencias en educación, y el informe sobre la formación profesional a distancia en España realizado por Sáez (2010); es decir, llevamos a cabo un análisis de contenido (Bardín, 1986) de las páginas web oficiales y del documento elaborado por Sáez (2010).

\section{Procedimiento utilizado para la Fase 2}

En la Fase 2 se analizó la información sobre el modelo organizativo a nivel institucional, y las herramientas tecnológicas y pedagógicas empleadas por los centros públicos que ofertan estudios a distancia de formación profesional. Para ello, se creó un cuestionario "ad hoc" compuesto por 15 ítems que servía como base para alcanzar dos objetivos: 1) verificar los datos recogidos en la Fase 1, o bien modificarlos en caso que estos estuvieran desactualizados o fuesen erróneos, y 2) recoger la opinión de una serie de expertos para dicho análisis.

Para la validación del cuestionario se utilizó la técnica de panel de expertos sobre una serie de aspectos significativos que nos facilitaran comprender el tipo de implantación de la FPaD que se estaba llevando a cabo en nuestras Comunidades Autonómicas, a partir de un proceso que constaba de dos vueltas:

- Para la construcción del cuestionario partimos de una primera versión realizada por nosotros que estaba compuesto de 15 ítems y que le fue suministrado en línea a expertos en el ámbito de la FPaD de cada Comunidad Autónoma (un experto por comunidad), siguiendo el orden alfabético por el nombre de la Comunidad Autónoma. Se mantuvo contacto por correo electrónico con todos y cada uno de los expertos y, una vez el experto analizaba el cuestionario y realizaba las aportaciones que considerase oportunas a la propuesta, se pasaba la nueva versión del cuestionario con las aportaciones realizadas al siguiente experto para recoger la opinión que tenía del mismo. Tras el envío de las propuestas de cuestionario a todas las Comunidades Autónomas, se obtuvo un cuestionario final con 29 ítems, que estaban clasificados en 7 bloques: institucionales (2 ítems), personales (3 ítems), organización institucional (4 ítems), plataforma educativa ( 5 ítems), materiales didácticos ( 7 ítems), profesorado (5 ítems) y recursos (2 ítems). El ítem número 29 sirvió de contenedor de sugerencias. La versión definitiva utilizada del cuestionario puede observarse en el Anexo del presente artículo.

- La versión final del cuestionario fue administrada de nuevo a todos los expertos, esta vez con el ruego de que lo cumplimentaran.

- Como han señalado Barroso y Cabero (2010) uno de los problemas que presenta la utilización de expertos es que su término es bastante polisémico, y por 
tanto en su utilización es aconsejable indicar con claridad qué ha entendido el investigador por ellos. Señalar que en nuestro trabajo los expertos eran profesores y profesoras que imparten o han impartido docencia en formación profesional en modalidad a distancia o semipresencial, así como personas especialistas con cargos organizativos en esta modalidad de formación. La combinación de ambas estrategias, análisis de los sitios web oficiales y la recogida de la información de diferentes expertos existentes en las distintas CC.AA, nos garantizaba poder crear un mapa y hacer una radiografía del estado de la cuestión de la FPaD que se estaba desarrollando en nuestro país, como base para futuras investigaciones. Con tales estrategias de recogida de información, se pudieron obtener datos respecto al número de matriculados en esta modalidad formativa, plataformas utilizadas, existencia de coordinadores, tipos de materiales y formatos utilizados,...; aspectos sobre los que posteriormente se presentarán algunos de los hallazgos encontrados.

\section{Procedimiento para la Fase 3}

La Fase 3 consistió en diseñar un mapa virtual interactivo bajo la premisa que se permitiera un acceso libre, neutral y transparente. Para conseguir que el acceso al mapa cumpliese esas tres características, se buscaron diferentes soluciones y, finalmente, se decidió utilizar la herramienta OpenStreetMap, que es un proyecto encargado de crear mapas libres y editables. Toda la cartografía está disponible bajo una licencia Creative Commons Reconocimiento-CompartirIgual 2.o (CC-BY-SA). Es decir, los mapas pueden copiarse, distribuirse, transmitirse y adaptarse libremente siempre y cuando se incluya una nota de reconocimiento a OpenStreetMap y a sus colaboradores. Si se altera o genera nuevo contenido sobre el mapa, estos cambios solo se podrán distribuir bajo la misma licencia.

Además de la creación del mapa, también fue necesario añadir la información correspondiente a los datos recogidos en el estudio. En base a cumplir la misma premisa de un acceso libre, neutral y transparente, se eligió Leaflet como herramienta de creación de contenidos para el mapa.

\section{POBLACIÓN Y MUESTRA DE ESTUDIO}

La población del presente estudio estaba formada por el conjunto de elementos que han sido expuestos en la tabla 1 y que se encuentran clasificados dentro de las dimensiones pedagógica, tecnológica y organizativa de la FPaD en las 17 Comunidades Autónomas españolas.

Para el análisis de las características de cada uno de los elementos, pudimos contactar con 17 expertos, cada uno de una Comunidad Autónoma, para que participara en primer lugar en la construcción conjunta del instrumento y en segundo 
lugar en la cumplimentación de la versión final del cuestionario. El cuestionario fue administrado en línea a toda la población y para ello se utilizó la herramienta Google Forms ${ }^{2}$.

La muestra obtenida fue del cien por cien de la población, ya que pudimos localizar y analizar las 17 páginas web oficiales destinadas a la FPaD dentro de los sitios web de las diferentes Consejerías de Educación y también recibimos las 17 respuestas de los expertos con toda la información requerida en el cuestionario suministrado a cada uno de ellos.

El estudio se llevó a cabo durante el curso académico 2014/2015.

\section{RESULTADOS}

\section{Datos de matrícula}

Para el análisis de los datos de matrícula en enseñanzas de FPaD, se consultaron las estadísticas que aparecen en la página Web del Ministerio de Educación, Cultura y Deporte (http://www.mecd.gob.es). A partir de los datos recogidos, se elaboró la figura 1, que muestra la evolución del número de matrículas en FPaD por grado formativo comprendidas desde su aparición en el curso 1993/1994 hasta la actualidad (curso 2014/2015).

Figura 1. Evolución de matriculaciones en la FPaD por grado formativo

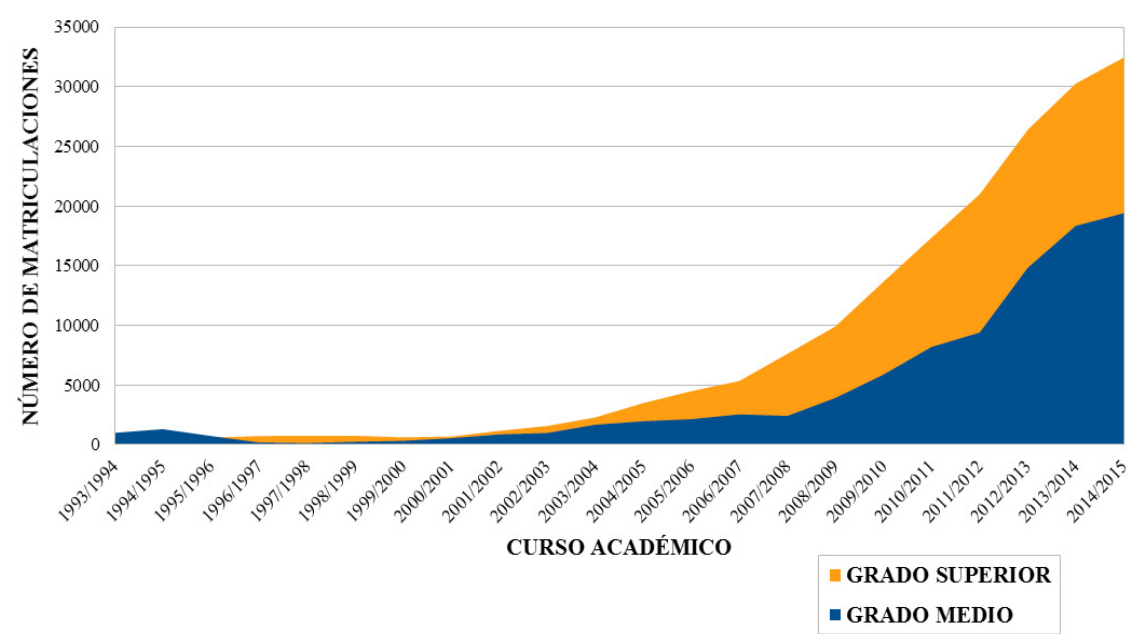




\section{Dimensión pedagógica}

El primer elemento que se analizó para la dimensión pedagógica fue el material didáctico. En concreto, se quería conocer si los centros educativos utilizan materiales diseñados específicamente para la impartición de las enseñanzas de FPaD y, en caso afirmativo, identificar algunas de sus características, que a continuación detallamos:

- Autoría. Persona, grupo u organización que ha producido el material didáctico.

- Herramienta de autor. Aplicación informática utilizada para la creación del material didáctico.

- Acceso universal. Variable dicotómica que responde a la pregunta de si el acceso al material didáctico es universal, es decir, si no existe limitación de ningún tipo para acceder a él.

- Tipo de licencia. Elección de la licencia de uso para el material didáctico, a partir de una clasificación simple de dos tipos: libre o propietaria.

- Formato de publicación. Tipo de formato utilizado para la difusión, clasificado en base a las siguientes características: puede ser modificado (editable), no puede ser modificado (no editable) o bien hay ciertas partes que pueden ser modificadas y otras no (mixto).

- Revisión periódica. Variable dicotómica que responde a la pregunta de si existen mecanismos de actualización del material didáctico.

En el cuadro 2 se muestran los resultados de la información recogida acerca de la creación y uso de materiales didácticos por Comunidades Autónomas.

Cuadro 2. Características básicas de los materiales didácticos en la FPaD

\begin{tabular}{|c|c|c|c|c|c|c|c|c|c|c|c|c|c|c|c|c|c|}
\hline \multirow[b]{2}{*}{$\begin{array}{c}\text { RASGO } \\
\text { BÁSICO } \\
\text { DEL } \\
\text { MATERIAL }\end{array}$} & \multicolumn{17}{|c|}{ COMUNIDAD AUTÓNOMA } \\
\hline & 苞 & 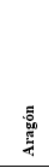 & 尊 & 离 & हू. & $\begin{array}{l}\text { हूँ } \\
\text { हूँ } \\
\text { हूँ }\end{array}$ & 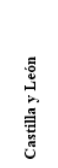 & 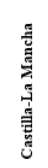 & 莺 & 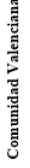 & 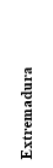 & 苞 & 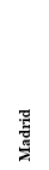 & 总 & 吾 & 总 & 苟 \\
\hline $\begin{array}{l}\text { Uso } \\
\text { especifico }\end{array}$ & $\mathrm{si}$ & $\mathrm{si}$ & No & $\mathrm{si}$ & $\mathrm{Si}$ & $\mathrm{Si}$ & $\mathrm{Si}$ & Si & $\mathrm{si}$ & No & $\mathrm{Si}$ & $\mathrm{si}$ & $\mathrm{Si}$ & $\mathrm{si}$ & $\mathrm{Si}$ & $\mathrm{si}$ & Si \\
\hline Autoria & MECD & MECD & - & IOC & MECD & MECD & $\begin{array}{l}J_{\text {unta }} \\
C y L\end{array}$ & MECD & Ioc & - & MECD & MECD & MECD & MECD & MECD & TKNIRA & MECD \\
\hline $\begin{array}{l}\text { Herramienta } \\
\text { de autor }\end{array}$ & eXe & exe & - & - & exe & exe & eXe & eXe & Wiki & - & eXe & eXe & exe & exe & exe & eXe & exe \\
\hline $\begin{array}{l}\text { Acceso } \\
\text { universal }\end{array}$ & No & No & - & $\mathrm{Si}$ & No & No & No & No & $\mathrm{Si}$ & - & No & No & No & No & No & No & No \\
\hline $\begin{array}{l}\text { Tipo de } \\
\text { licencia }\end{array}$ & Libre & Libre & - & Libre & Libre & Libre & Propieterria & Libre & Libre & - & Libre & Libre & Libre & Libre & Libre & Libre & Libre \\
\hline $\begin{array}{l}\text { Formato de } \\
\text { publicación }\end{array}$ & Minto & Misto & - & $\begin{array}{c}\text { No } \\
\text { editable }\end{array}$ & Minto & Minto & Editable & Mirto & $\begin{array}{c}\text { No } \\
\text { editable }\end{array}$ & - & Minto & Minto & Minto & Minto & Misto & Misto & Minto \\
\hline $\begin{array}{l}\text { Revisión } \\
\text { periódica }\end{array}$ & $\mathrm{Si}$ & No & - & No & $\mathrm{Si}$ & $\mathrm{Si}$ & Si & $\mathrm{Si}$ & $\mathrm{si}$ & - & No & No & No & $\mathrm{Si}$ & No & Si & No \\
\hline
\end{tabular}


El segundo elemento analizado para la dimensión pedagógica corresponde con el uso de herramientas de comunicación e interacción. Se comprobó que las herramientas son comunes en todas las Comunidades Autónomas, tanto en modalidad asíncrona (los foros y los mensajes), como en modalidad síncrona (el chat).

El tercer elemento a analizar para la dimensión pedagógica estaba compuesto por dos parámetros: a) el tamaño de la clase, entendiendo como tal el número máximo de discentes por cada docente y b) la carga de trabajo, es decir, el número de horas que el docente tiene asignadas para la dedicación a la tutoría por cada módulo formativo. Para facilitar la interpretación de los datos, en la figura 2 se muestra la relación entre estas dos variables (que hemos denominado horas de dedicación docente al estudiante) por horas semanales de un módulo. Como guía de referencia se ha empleado la media sobre la ratio existente en los estudios de enseñanza presencial, es decir, las horas semanales de dedicación docente al estudiante por módulo formativo para un aula de 30 estudiantes.

Figura 2. Dedicación docente al estudiante por horas semanales del módulo formativo ${ }^{3}$

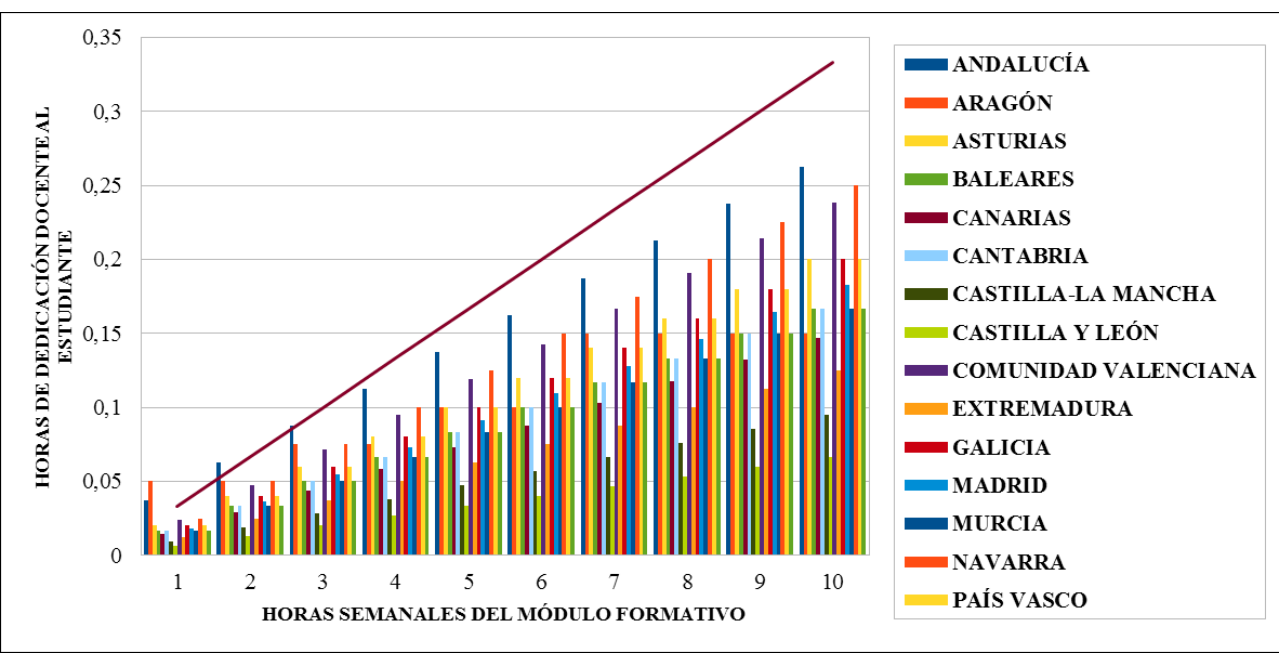

\section{Dimensión tecnológica}

El primero de los elementos analizados para la dimensión tecnológica fue el tipo de plataforma EVA utilizado para impartir los estudios de FPaD. Se confirmó que Moodle es la plataforma empleada para todo el territorio español. A partir del mapa virtual interactivo que se ha creado en el presente estudio http://www. cristiangarcia.org/mapaFPaD.htm] se puede acceder a cada una de las páginas web 
oficiales de la plataforma EVA que se emplea en las Comunidades Autónomas para impartir las enseñanzas de FPaD.

El segundo de los elementos que se analizaron para la dimensión tecnológica fueron los recursos y servicios que cada institución suministra al alumnado. Además, en el caso de que el recurso o servicio lo permitiera, también se analizó si este se ponía a disposición del alumnado para que lo pudiera utilizar fuera de las instalaciones del centro educativo. La figura 3 muestra los resultados obtenidos para este elemento.

Figura 3. Recursos y servicios disponibles en la FPaD por Comunidad Autónoma

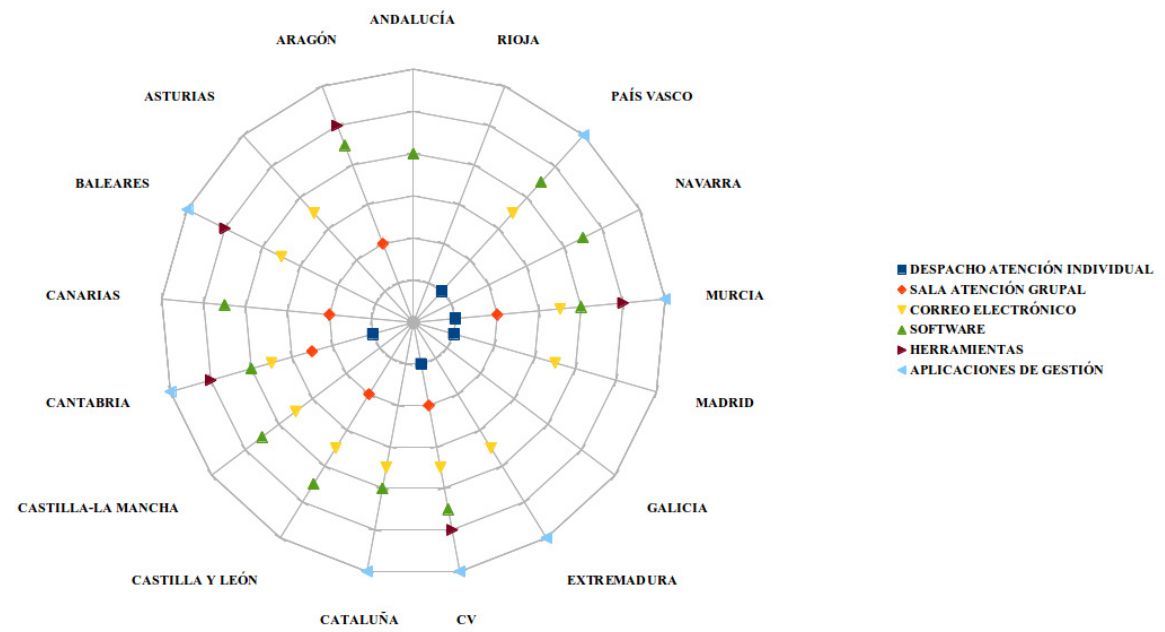

\section{Dimensión organizativa}

Para finalizar, en la dimensión organizativa se trataron los aspectos institucionales que afectan a la implementación y el desarrollo de las enseñanzas a distancia de formación profesional. El primer elemento que se analizó en esta dimensión fue el modelo de organización que se emplea en cada Comunidad Autónoma a nivel institucional. Debido a que existen similitudes entre varios de los modelos organizativos analizados, se ha elaborado una clasificación que los sintetiza en tres tipos genéricos:

- Modelo Tipo I. La oferta de estudios a distancia de formación profesional es asumida por los centros públicos que imparten enseñanzas presenciales. La dedicación por parte del profesorado a la impartición y a la coordinación no es exclusiva para la formación a distancia. Es el caso más extendido y se emplea en todas las Comunidades Autónomas, excepto Cataluña y País Vasco. 
- Modelo Tipo II. La oferta formativa se centraliza en una única sede con profesorado propio, aunque se dispone de centros colaboradores repartidos por toda la Comunidad Autónoma, en los que se ofrecen los servicios básicos de: a) recogida de documentación durante los procesos de matrícula y b) cesión de instalaciones para la organización de actividades presenciales, como por ejemplo pruebas de evaluación final o jornadas técnicas. Cataluña utiliza este modelo organizativo.

- Modelo Tipo III. Existe un único centro que se encarga de ofertar y gestionar la formación a distancia de toda la Comunidad Autónoma.

También se dispone de una red de centros de referencia, del que hay uno por ciclo formativo. En cada uno de los centros de referencia se seleccionan tanto el equipo docente como los coordinadores de ciclo, que se dedican exclusivamente a la formación a distancia, aunque físicamente desempeñan sus funciones en su centro de procedencia.

Además, existen los llamados centros colaboradores, de los que hay uno por ciclo en cada provincia. Los docentes de estos centros, coordinados por el docente titular del módulo formativo, se encargan exclusivamente de llevar a cabo las sesiones presenciales de cada módulo. Es decir, los docentes de los centros colaboradores no pertenecen a la estructura de la docencia a distancia, pero colaboran durante determinadas horas en la impartición de las sesiones presenciales. Este modelo es el utilizado en el País Vasco.

Dentro del modelo Tipo I se puede realizar una división a partir del número de centros que imparten un mismo ciclo, con lo que aparecen dos subtipos:

- Modelo Tipo I, formación especializada. Un ciclo formativo únicamente se imparte en un centro educativo para toda la Comunidad Autónoma. Es el submodelo empleado en Canarias, Cantabria, Ceuta, Extremadura, Melilla, Murcia, Navarra y Rioja.

- Modelo Tipo I, formación descentralizada. Un ciclo formativo puede impartirse en varios centros para toda la Comunidad Autónoma. Es el utilizado en Andalucía, Aragón, Asturias, Baleares, Castilla y León, CastillaLa Mancha, Comunidad Valenciana, Galicia y Madrid.

El segundo elemento analizado para la dimensión organizativa es la estrategia de implementación de las enseñanzas a distancia de formación profesional. En la tabla 1 aparecen la totalidad de ciclos formativos que se ofertan en modalidad a distancia durante el curso 2014/2015 y el número de centros donde se imparte cada uno de ellos para cada Comunidad Autónoma. 
Tabla 1. Número de centros con ciclos formativos ofertados en la FPaD por C. Autónoma en el curso 2014/2015

\begin{tabular}{|c|c|c|c|c|c|c|c|c|c|c|c|c|c|c|c|c|c|c|c|}
\hline \multirow{2}{*}{\multicolumn{2}{|c|}{$\begin{array}{l}\text { FAMILIA PROFESIONAL Y } \\
\text { CICLO FORMATIVO } \\
\text { Andalucía }\end{array}$}} & \multicolumn{18}{|c|}{ COMUNIDAD AUTÓNOMA } \\
\hline & & $\begin{array}{l}7 \\
0 \\
\infty \\
0 \\
3 \\
3 \\
4\end{array}$ & $\frac{\frac{\pi}{0}}{\frac{\pi}{3}}$ & 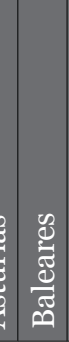 & 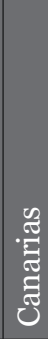 & 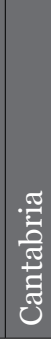 & 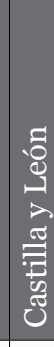 & 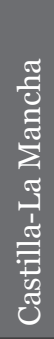 & 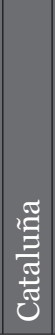 & $\begin{array}{l} \\
\stackrel{\pi}{3} \\
0 \\
0\end{array}$ & 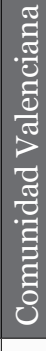 & 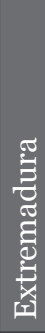 & : & 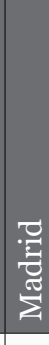 & $\mid \stackrel{\varpi}{\stackrel{\Xi}{:}}$ & $\begin{array}{l}\frac{\pi}{0} \\
ٍ \\
\Xi \\
\end{array}$ & 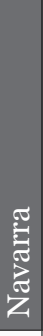 & 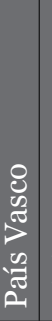 & $\frac{\pi}{2}$ \\
\hline \multirow{3}{*}{$\begin{array}{l}\text { Administración y } \\
\text { gestión }\end{array}$} & $G A$ & & 3 & 1 & 3 & 1 & 1 & 10 & 4 & 1 & & 2 & 1 & 2 & 7 & & 1 & & \\
\hline & $A F$ & 1 & 1 & & 3 & 1 & & & 3 & 1 & & 4 & 1 & 3 & & & 1 & & \\
\hline & $A D$ & 1 & 1 & & 3 & & & & & 1 & & & & & & & & & \\
\hline \multirow{4}{*}{ Agraria } & $P A E$ & & & & & & 1 & & & & & & & & & & & & \\
\hline & $P A P$ & & & & & 1 & 1 & & & & & & & & & & & & \\
\hline & GFMN & & & & & & & & & & & 1 & & & & & & & \\
\hline & PMR & & & & & & & & & & & 1 & & & & & & & \\
\hline \multirow{8}{*}{$\begin{array}{l}\text { Comercio y } \\
\text { marketing }\end{array}$} & $A C$ & & & 1 & & & & & & & & 1 & & & & & & & \\
\hline & $C I$ & & 1 & & & & & 2 & 2 & 1 & & 3 & & & & & & & \\
\hline & $C I^{2}$ & & 1 & & & & & & & & & & & & & & & & \\
\hline & $G C M^{2}$ & & 1 & & & & & & & 1 & & & 1 & & & & & & \\
\hline & GVEC & 1 & 1 & 1 & & 1 & & 1 & 2 & 1 & & 1 & & & & & & & \\
\hline & $G T$ & & & & & & & & & & & & 1 & & & & & & \\
\hline & $M P$ & & & & & & & & 1 & & & & & & & & & 1 & \\
\hline & $T L$ & 1 & 1 & 1 & & & 1 & 1 & 1 & & & & & 1 & & & & & \\
\hline $\begin{array}{l}\text { Edificación y } \\
\text { obra civil }\end{array}$ & $P E$ & & & & & & & & & & & 1 & & 1 & & & & & \\
\hline \multirow{5}{*}{$\begin{array}{l}\text { Electricidad y } \\
\text { electronica }\end{array}$} & $I T$ & & & & & & & & & & & 3 & & 1 & & & & & \\
\hline & $I E A$ & & 1 & 1 & 3 & 1 & 1 & 2 & 1 & 1 & & 6 & 1 & 1 & & & 1 & & 1 \\
\hline & $A R I$ & & & & & & & & & & & 2 & & & & & & 1 & \\
\hline & $S T I$ & & & & & 1 & & & & & & 3 & & 1 & & & & & \\
\hline & SEA & & & & & 1 & 1 & & & & & 2 & & & & & & & \\
\hline \multirow{2}{*}{ Energía y agua } & $C E$ & & & & & & & & & & & & & & & & & 1 & \\
\hline & EEEST & & & & & 1 & 1 & & & & & & & & & & & & \\
\hline \multirow{3}{*}{$\begin{array}{l}\text { Fabricación } \\
\text { mecánica }\end{array}$} & $S C$ & & & & & & 1 & & & & & & & & & & & & \\
\hline & $D F M$ & & & & & & & & & & & & & & & & & & 1 \\
\hline & $P P F M$ & & & & & & & & & & & & & & & & & & 1 \\
\hline
\end{tabular}




\begin{tabular}{|c|c|c|c|c|c|c|c|c|c|c|c|c|c|c|c|c|c|c|c|}
\hline \multirow{2}{*}{\multicolumn{2}{|c|}{$\begin{array}{l}\text { FAMILIA PROFESIONAL Y } \\
\text { CICLO FORMATIVO } \\
\text { Andalucía }\end{array}$}} & \multicolumn{18}{|c|}{ COMUNIDAD AUTÓNOMA } \\
\hline & & 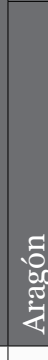 & $\mid \begin{array}{l}\frac{\pi}{0} \\
\frac{\pi}{3} \\
\frac{3}{3} \\
\frac{3}{4}\end{array}$ & 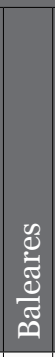 & 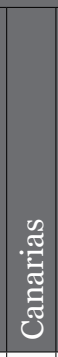 & 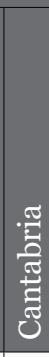 & 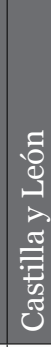 & 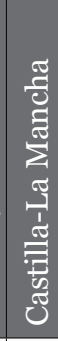 & 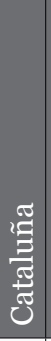 & త্ & 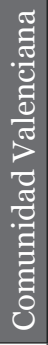 & 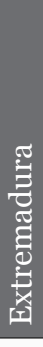 & 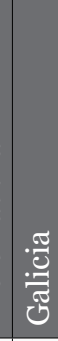 & 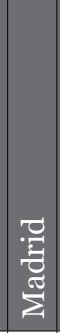 & 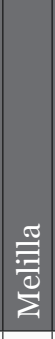 & $\begin{array}{l}\frac{\sigma}{0} \\
\vdots \\
\vdots \\
\Sigma\end{array}$ & 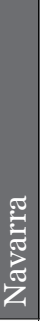 & 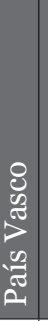 & \%) \\
\hline \multirow{7}{*}{$\begin{array}{l}\text { Hostelería y } \\
\text { turismo }\end{array}$} & $C G$ & & & 1 & 3 & 1 & 1 & & & & 1 & & & & & & & & 1 \\
\hline & $S R$ & & & 1 & & & & & & & & & & & & & & & \\
\hline & $A V G E$ & 1 & 1 & & 3 & & & & & & & 1 & & 1 & & & & & \\
\hline & $D C$ & & & & & 1 & & & & & & & & 1 & & & & & \\
\hline & $D S R$ & & & & & 1 & & & & & & & & & & & & & \\
\hline & $G A T$ & & & 1 & 3 & & & & & & & 1 & & 1 & & & & 1 & \\
\hline & GIAT & 1 & & & 3 & & & & & & & 1 & & 1 & & & & & \\
\hline \multirow{3}{*}{ Imagen personal } & $E B$ & & & & & 1 & & & & & & & & & & & & & \\
\hline & $P C C$ & & & & & 1 & & & & & & 1 & & & & & & & \\
\hline & $E I B$ & & & & & & & & & & & 1 & & & & & & & \\
\hline \multirow{4}{*}{ Imagen y sonido } & $V D S$ & & & & & & & & & & & 2 & & & & & & & \\
\hline & ICTI & & & & & & & & & & & 1 & & & & & & & \\
\hline & $P A E$ & & 1 & & & & & & & & & & & 1 & & & & & \\
\hline & $S A E$ & & & & & & & & & & & & & 1 & & & & & \\
\hline \multirow{3}{*}{$\begin{array}{l}\text { Industrias } \\
\text { alimentarias }\end{array}$} & $P R C$ & & & & 3 & 1 & & & & & & & & 1 & & & & & \\
\hline & PCIA & & & & & & 1 & & & & & & & & & & & & \\
\hline & $V V$ & & & & & & & & & & & & & & & & & & \\
\hline \multirow{4}{*}{$\begin{array}{l}\text { Informática y } \\
\text { comunicaciones }\end{array}$} & $S M R$ & & 1 & 1 & 3 & 1 & 1 & 1 & 4 & 1 & & 4 & & 1 & & & & 1 & \\
\hline & ASIR & & 1 & 1 & 3 & 1 & 1 & 1 & & 1 & & 4 & 1 & 1 & & & & 1 & 1 \\
\hline & $D A M$ & 1 & 1 & 2 & & 1 & 1 & 2 & 3 & 1 & & 5 & 1 & 1 & & & 1 & & \\
\hline & $D A W$ & 1 & 1 & 2 & & 1 & 1 & 2 & 1 & 1 & & 8 & 1 & 2 & & & 1 & 1 & \\
\hline & $I P C$ & & & 1 & & & & & & & & 1 & & & & & & & \\
\hline & $I F C$ & & & 1 & & & & & & & & 2 & & & & & 1 & & \\
\hline Instalación y & $M E$ & & & & & & & & & & & 2 & & & & & & & \\
\hline mantenimiento & DPITF & & & 1 & & & & & & & & & & & & & & & \\
\hline & $M I$ & & & & & & 1 & & & & & 2 & & & & & & & \\
\hline & PRP & & & 1 & & & & & 1 & & & & 1 & & & & & & \\
\hline $\begin{array}{l}\text { Madera, mueble } \\
\text { y corcho }\end{array}$ & $D A$ & & & & & & & & & & & & & 1 & & & 1 & & \\
\hline
\end{tabular}




\begin{tabular}{|c|c|c|c|c|c|c|c|c|c|c|c|c|c|c|c|c|c|c|c|c|}
\hline \multirow{2}{*}{\multicolumn{2}{|c|}{$\begin{array}{l}\text { FAMILIA PROFESIOI } \\
\text { CICLO FORMATIVO } \\
\text { Andalucía }\end{array}$}} & \multicolumn{19}{|c|}{ COMUNIDAD AUTÓNOMA } \\
\hline & & \multirow[t]{2}{*}{ 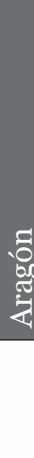 } & \multirow[t]{2}{*}{$\frac{\sqrt[c]{3}}{\frac{0}{3}}$} & \multirow[t]{2}{*}{ 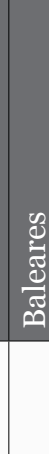 } & \multirow[t]{2}{*}{ - } & \multirow[t]{2}{*}{ 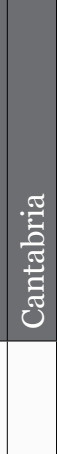 } & \multirow[t]{2}{*}{ 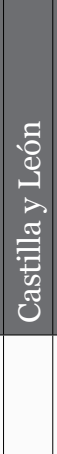 } & \multirow{2}{*}{\multicolumn{2}{|c|}{ 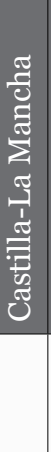 }} & \multirow[t]{2}{*}{ 䄈| } & \multirow[t]{2}{*}{ 氶 } & 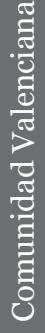 & \multirow[t]{2}{*}{ 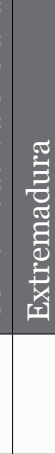 } & \multirow[t]{2}{*}{ 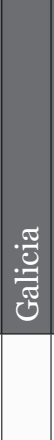 } & \multirow[t]{2}{*}{ 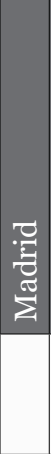 } & \multirow[t]{2}{*}{ 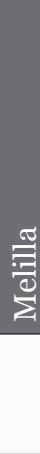 } & \multirow[t]{2}{*}{$\begin{array}{c}\frac{\pi}{0} \\
\frac{\pi}{3} \\
\sum\end{array}$} & \multirow[t]{2}{*}{ 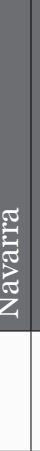 } & 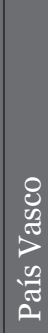 & \multirow[t]{2}{*}{ 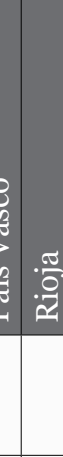 } \\
\hline $\begin{array}{l}\text { Mantenimiento } \\
\text { de vehículos } \\
\text { autopropulsados }{ }^{4}\end{array}$ & $E V A$ & & & & & & & & & & & 1 & & & & & & & & \\
\hline $\begin{array}{l}\text { Mantenimiento } \\
\text { y servicios a la } \\
\text { producción }{ }^{4}\end{array}$ & $P R P$ & 2 & & & & & & & & & 1 & & 2 & & 1 & & & & & \\
\hline Química & $L A C C$ & & 1 & & & & & & & & & & & & 1 & & & 1 & & \\
\hline \multirow{7}{*}{ Sanidad } & $C A E$ & & & 3 & & 1 & & & & & 1 & & 5 & & & & & & & \\
\hline & $E S$ & & 2 & 1 & 3 & 1 & 1 & 2 & & & 1 & 1 & 4 & 1 & 2 & & & 1 & 1 & 1 \\
\hline & $F P$ & & 1 & & 3 & 1 & 1 & & & & & & & & 1 & & & & & \\
\hline & $D$ & & & & & 1 & & & & & & & 1 & & & & & & & \\
\hline & $D S$ & & & & & & & & & & & & 2 & & & & & & & \\
\hline & $I D$ & & & & & & & & & & & & 1 & & & & & & & \\
\hline & $O P$ & & & & & & & & & & & & 1 & & & & & & & \\
\hline $\begin{array}{l}\text { Seguridad y } \\
\text { medio ambiente }\end{array}$ & $E C A$ & & & & & & & & & & & & 1 & & 1 & & & & & \\
\hline \multirow{4}{*}{$\begin{array}{l}\text { Servicios } \\
\text { socioculturales y } \\
\text { a la comunidad }\end{array}$} & $A P S D$ & 1 & 2 & 2 & 3 & 1 & 1 & & & & 1 & & 7 & & 3 & & & 1 & & 1 \\
\hline & $A S T$ & & & & & & & & & & & & 1 & & & & & & & \\
\hline & $E I$ & 1 & 2 & 2 & 3 & 1 & 1 & 5 & & & 1 & 1 & 3 & 1 & 2 & 5 & 1 & 1 & 1 & 1 \\
\hline & $I S$ & & 1 & & & 1 & & & & & 1 & & 5 & & & & & & & \\
\hline \multirow{3}{*}{$\begin{array}{l}\text { Transporte y } \\
\text { mantenimiento } \\
\text { de vehículos }\end{array}$} & $C$ & & & & & & & & & & & & 1 & & & & & & & \\
\hline & $E V A$ & & & & & 1 & 1 & & & & & & 1 & & & & & & & \\
\hline & $A$ & & & & & & & & & & & & 2 & & & & & & & \\
\hline
\end{tabular}

\section{Mapa interactivo}

El mapa interactivo creado en la Fase 3 como parte de los objetivos del presente estudio (accesible desde http://www.cristiangarcia.org/mapaFPaD.html) presenta todas y cada una de las páginas web oficiales de la plataforma EVA que se emplean en las Comunidades Autónomas para impartir las enseñanzas de FPaD. En la imagen 
1 se muestra una captura con el mapa virtual en el que se ha ejemplificado el acceso al EVA utilizado en Andalucía.

Imagen 1. Captura de imagen del mapa virtual interactivo con un ejemplo del EVA utilizado en Andalucía

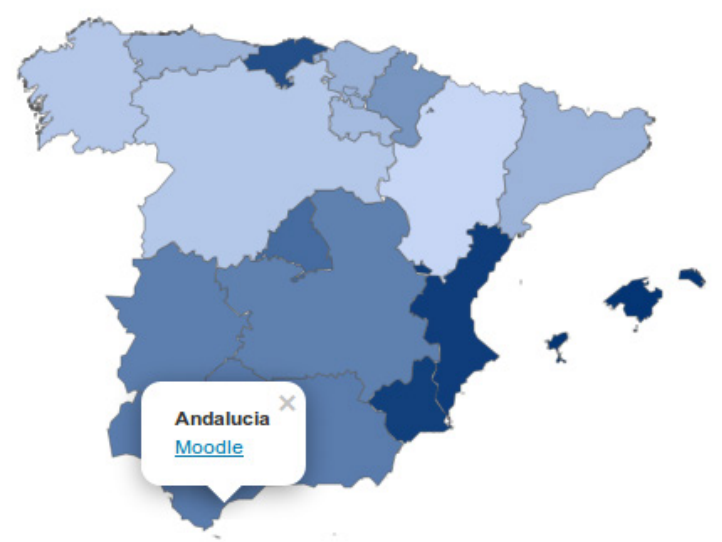

\section{CONCLUSIONES}

En primer lugar, se ha constatado un aumento continuo en el número de estudiantes matriculados en enseñanzas a distancia de formación profesional, desde su aparición en el curso 1993/1994, en el que se registraron un total de 1229 matrículas, hasta el curso actual 2014/2015 en el que se han registrado un total de 21855 matrículas. Tendencia que creemos que irá progresivamente en aumento, y que confirma las expectativas propuestas por diferentes autores, cuando nos señalan que la educación a distancia se está convirtiendo en una educación disruptiva (García 
Aretio, 2014) y en una modalidad de formación que potencia la inclusión social y educativa (Cabero, 2015).

En segundo lugar, los resultados sobre el análisis de los materiales didácticos muestran que gran parte de las Comunidades Autónomas (Andalucía, Aragón, Canarias, Cantabria, Castilla-La Mancha, Extremadura, Galicia, Madrid, Murcia, Navarra y Rioja) utilizan materiales didácticos elaborados por el Ministerio de Educación, Cultura y Deporte específicamente para las enseñanzas de FPaD; por su parte, Castilla y León, Baleares, Cataluña y País Vasco también utilizan materiales didácticos específicos para la FPaD, aunque están elaborados por la Junta de Castilla y León, por el Institut Obert de Catalunya (IOC) y por el Centro de Innovación para la Formación Profesional (TKNIKA), respectivamente; por lo que respecta a la Comunidad Valenciana y Asturias, son comunidades que no emplean materiales didácticos diseñados específicamente para los estudios de FPaD. Los resultados también muestran que, si bien la mayoría de Comunidades Autónomas utilizan unos materiales específicos para la $\mathrm{FPaD}$, estas no prevén actualizaciones de ningún tipo, razón por la que los materiales pueden quedar obsoletos y, por tanto, carentes de utilidad alguna. Lo expuesto, nos lleva a sugerir que sería necesario: a) controlar la actualización de los materiales mediante una serie de mecanismos como por ejemplo son el uso de formatos de contenido editables, la publicación del contenido bajo licencias libres y la gestión de revisiones sobre un espacio común y de acceso universal, y b) llevar a cabo diferentes investigaciones en una doble dirección, por una parte centradas en analizar la fundamentación conceptual en la cual se apoyan las instituciones para la producción de los materiales, y por otra para analizar cómo implementar adecuadamente estos mecanismos para la creación y la revisión de contenidos en las enseñanzas de FPaD. Tales estudios se hacen muy necesarios en la $\mathrm{FPaD}$, si tenemos en cuenta las especificidades de este tipo de estudios y la significación que tienen los materiales de enseñanza en esta modalidad de formación (Cabero y Gisbert, 2005; García Aretio, 2014).

En tercer lugar, todas y cada una de las Comunidades Autónomas han elegido la plataforma Moodle como entorno virtual de aprendizaje (EVA) para la impartición de sus estudios a distancia de formación profesional. Este dato nos revela la importancia que está adquiriendo la plataforma Moodle en las enseñanzas de modalidad a distancia, cuyo índice de implantación está aumentando de manera progresiva en todo el mundo. Dato en el cual nuestro estudio coincide con lo que está ocurriendo en otros niveles educativos como el universitario (Gómez Rey, Hernández García, Rico García, 2009; Martín Galán, Rodríguez Mateos, 2012; Marín, Ramírez y Sampedro, 2011; Mirabal, Gómez y González, 2015).

Aunque el hecho de elegir una plataforma en concreto no determine una mejora en los resultados del proceso de enseñanza-aprendizaje, sí que puede ayudar para poder sacar mayor partido a cualquier mecanismo de innovación que venga determinado por el uso de una plataforma EVA, como por ejemplo sería la implementación de algunos artefactos TIC en concreto, ya que dicho mecanismo 
podría aplicarse a todo el ámbito nacional sin necesidad de realizar adaptaciones a otras plataformas EVA. También como parte de los resultados obtenidos en el estudio, desde la dirección web http://www.cristiangarcia.org/mapaFPaD.htm se encuentra un mapa interactivo que permite el acceso a cada una de las páginas web oficiales de la plataforma EVA que se emplean en las Comunidades Autónomas para impartir las enseñanzas de FPaD.

En cuarto lugar, y respecto al tamaño de las aulas, la ratio del número máximo de estudiantes por módulo formativo varía entre Comunidades Autónomas, comenzando desde 40 estudiantes por módulo (la menor ratio entre todas las comunidades) para el caso de Aragón y Navarra, y alcanzando un total de 100 estudiantes por módulo (la mayor ratio de todas las comunidades) en los casos de Andalucía y Cataluña. Además, la carga horaria que el profesorado tiene asignada para la acción tutorial también es diferente entre las Comunidades Autónomas. En comparación con la carga lectiva que tiene un módulo formativo en los estudios presenciales ( $\mathrm{N}$ horas), se llega a duplicar las horas en la comunidad que más carga destina a la acción tutorial (Andalucía, con $2 \mathrm{~N}+1$ horas por módulo formativo) y a dividir las horas entre dos en la comunidad que menos horas destina a ella (Castilla y León, con N/2 horas por módulo). Al cruzar la información sobre el tamaño de aula y la carga horaria, los resultados muestran que las horas de dedicación docente en la modalidad FPaD en ningún caso se equiparan a sus equivalentes en la modalidad presencial. Únicamente cabría destacar el hecho de que algunas Comunidades Autónomas, como Cataluña y Andalucía, se esfuerzan por utilizar fórmulas que equilibren ambos parámetros. Si tenemos en cuenta la especial dificultad que entraña esta modalidad de formación tanto para el alumnado como para el profesorado, sería deseable utilizar los mecanismos necesarios, como por ejemplo el empleo de fórmulas específicas, para que al menos se consiguieran ratios de dedicación docente al estudiante, parecidos a los obtenidos en las enseñanzas de modalidad presencial.

En quinto lugar, en lo referente a la organización a nivel institucional, se ha constatado que la oferta de enseñanzas a distancia de formación profesional es asumida por los profesores de los centros públicos que imparten enseñanzas presenciales en la práctica totalidad de Comunidades Autónomas, a excepción de Cataluña y País Vasco. En Cataluña, la formación a distancia se lleva a cabo por profesorado propio desde una única sede central ubicada en Barcelona, el Instituto Abierto de Cataluña (IOC - Institut Obert de Catalunya), que recibe apoyo de los llamados centros colaboradores para ofrecer algunos de los servicios presenciales. En el caso del País Vasco, existe un único centro, el Instituto de Formación Profesional a Distancia (ULHI - Urrutiko Lanbide Heziketako Institutoa), encargado de la oferta y de la gestión de la formación a distancia, que incorpora como profesorado propio a los docentes de los llamados centros de referencia, aunque físicamente desempeñan sus funciones en su centro de procedencia; además, esta estructura organizativa también incluye la figura de los centros y profesores colaboradores, que ayudan en la impartición de las sesiones presenciales. 
En sexto lugar, tras haber analizado la oferta formativa de enseñanzas a distancia de FP en las diferentes Comunidades Autónomas, se constata que existe oferta para casi todas las familias profesionales, a excepción de las familias, tales como: actividades físicas y deportivas, artes gráficas, artes y artesanía, industrias extractivas, marítimo-pesquera, textil, confección y piel y vidrio y cerámica, de las que no existe ofertado ningún ciclo formativo.

Para finalizar, señalar que los datos encontrados nos indica la disparidad existente entre las diferentes Comunidades Autónomas, lógico por una parte al tener la transferencia de las competencias educativas. Por ello, sería deseable el establecer mecanismos de coordinación y de comunicación que ayudaran a la mejora en las tres dimensiones (pedagógica, tecnológica y organizativa) que han sido analizadas en el presente estudio. Por otra parte, se hace necesario el establecer una línea de investigación de las posibilidades que ofrece el e-learning en la formación profesional a distancia, ya que las administraciones se han dedicado a su puesta en funcionamiento pero no han invertido en estudios de evaluación ni en investigaciones para su perfeccionamiento.

\section{NOTAS}

1. http://blog.originlearning.com/10-facts-about-e-learning

2. https://www.google.com/forms/about

3. Cataluña no aparece en la figura debido a que el cálculo de la dedicación docente al estudiante no depende exclusivamente de las horas semanales del módulo formativo.

4. Estudios LOGSE (en extinción).

\section{REFERENCIAS BIBLIOGRÁFICAS}

Aguaded, J. I., y Cabero, J. (2013). Tecnologías y medios para la educación en la e-sociedad. Madrid: Alianza.

Area, M., y Adell, J. (2009). E-learning: enseñar y aprender en espacios virtuales. En J. De Pablos (coord.), La formación del profesorado en la era de internet. (391424). Málaga: Aljibe.

Bardín, L. (1986). Análisis de contenido. Madrid: Akal.

Barroso, J., y Cabero, J. (2010). La investigación educativa en TIC. Visiones prácticas. Madrid: Síntesis.

Bartolomé, A. (2004). Blended learning. Conceptos básicos. Pixel-Bit. Revista de medios y educación, 23, 7-20. Recuperado de
pixelbit/articulos/n23/PIXEL BIT 23. pdf

Cabero, J. (2006). Bases pedagógicas del e-learning. RUSC. Universities and knowledge society journal, 3(1). doi: http://dx.doi.org/10.7238/rusc.v3i1.265

Cabero, J. (2015). La educación a distancia como estrategia de inclusión social y educativa. Revista Mexicana de Educación a Distancia, 15. Recuperado de http:/ bdistancia.ecoesad.org. $m x /$ ?articulo=laeducacion-a-distancia-como-estrategiade-inclusion-social-y-educativa

Cabero, J., y Gisbert, M. (2005). La formación en Internet. Guía para el diseño de materiales didácticos. Sevilla: Eduforma. 
Díaz, M. D., Álvarez, E., y Rodríguez, A. (2013). Ecosistemas de formación autónomos en el desarrollo profesional del pedagogo. Pixel-Bit. Revista de medios y educación, 43, 99-112. doi: http://dx.doi. org/10.12795/pixelbit.2013.i43.05

Duart, J. M., y Lupiáñez, F. (2005). La perspectiva organizativa del e-learning. RUSC. Universities and knowledge society journal, 2(1). doi: http://dx.doi. org/10.7238/rusc.v2i1.242

Fundación Apel. (2013). El impacto de e-learning en el sistema de la Formación Profesional para el Empleo. Málaga: Vértice.

Dukes, L., Waring, S., y Koorland, M. (2006). The blended course delivery method. Journal of computing in teacher education 22(4), 153-158. Recuperado de http://www.tandfonline.com/doi/abs/10. 1080/10402454.2006.10784551

García Aretio, L. (2014). Bases, mediaciones y futuro de la educación a distancia en la sociedad digital. Madrid: Síntesis.

Gómez Rey, I., Hernández García, E., y Rico García, M. (2009). Moodle en la enseñanza presencial y mixta del inglés en contextos universitario. RIED. Revista Iberoamericana de Educación a Distancia, 12(1), 169-193. doi: http:// dx.doi.org/10.5944/ried.1.12.926

Khan, B. H. (2001). A framework for webbased learning. En B. H. Khan (Ed.), Web-based training (75-92). New Jersey: Englewood Cliffs.

Khan, B. H. (2015). Introduction to e-learning. En B. H. Khan y M. Ally (Eds.), International handbook of e-learning (140). London: Routledge.

Marín, V., Ramírez, A., y Sampedro, B. (2011). Moodle y estudiantes universitarios. Dos nuevas realidades del EEES. Profesorado. Revista de Currículum y formación del profesorado. 15(1). Recuperado de http:/ www.ugr.es/local/recfpro/rev151ART7. pdf
Martín, B., y Rodríguez, D. (2012). La evaluación de la formación universitaria semipresencial y en línea en el contexto del EEES mediante el uso de los informes de actividad de la plataforma Moodle. RIED. Revista Iberoamericana de Educación a Distancia, 15(1), 159-178, doi: http:/ dx.doi.org/10.5944/ried.15.1

Marsh, G. E., McFadden, A. C., y Price, B. J. (2003). Blended instruction: adapting conventional instruction for large classes. Online journal of distance learning administration 4(4). Recuperado de http://www.westga.edu/ distance/ojdla/ winter64/marsh64.htm

Mirabal, A., Gómez, M., y González, L. (2015). El uso de la plataforma Moodle como apoyo a la docencia presencial universitaria. EDMETC. Revista de Educación Mediática y TIC, 4(1), 133155. Recuperado de http://www.uco.es ucopress/ojs/index.php/edmetic/article/ download/2903/2831

Núñez, T. (2011). Entornos virtuales de enseñanza aprendizaje (EVEA): formación profesional. Edutec-e, revista electrónica de tecnología educativa, 37. Recuperado de http://www.edutec.es/revista/index. php/edutec-e/article/view/389

Sáez, J. (2010). Informe sobre la Formación Profesional a distancia en España. Organización, oferta, metodología y herramientas sw utilizadas. Parte 1a. Introducción y ámbito autonómico. Revista de Educación a Distancia, 25. Recuperado de http://revistas.um.es/red article/view/125291

Salinas, J. (2004). Cambios metodológicos con las TIC: estrategias didácticas $y$ entornos virtuales de enseñanzaaprendizaje. Recuperado de http://goo. gl/PGxNUS

Salinas, J. (2005). La gestión de los entornos virtuales de formación. Recuperado de http://gte.uib.es/pape/gte/sites/gte.uib. es.pape.gte/files/gestioEVEA o.pdf 
Sangrà, A. (2008). La integració de les TIC a la universitat: models, problemes $i$ reptes (Tesis doctoral no publicada). Recuperado de http://tdx.cbuc.es handle/10803/8947

Vega, N. (2013). Asesorías innovadoras en recursos humanos para las PYMES mediante el uso de la red de internet. Revista Nacional de Administración, 4(1), 85-100. Recuperado de http://investiga. uned.ac.cr/revistas/index.php/rna/ article/view/535

\section{PERFIL ACADÉMICO Y PROFESIONAL DE LOS AUTORES}

Cristian Jorge García Marcos. Ingeniero informático, Máster de software libre y Máster de Educación y TIC (e-learning) por la Universitat Oberta de Catalunya. Doctorando de la Facultad de Ciencias de la Educación de la Universidad de Sevilla en la línea de investigación las tecnologías de la información y comunicación en los ámbitos educativos. Profesor de educación secundaria en la especialidad de informática y colaborador docente en los estudios de informática, multimedia y telecomunicación de la Universitat Oberta de Catalunya.

E-mail: crigarmar9@alum.us.es

Julio Cabero Almenara. Catedrático de Tecnología Educativa de la Universidad de Sevilla, director del Secretariado de Recursos Audiovisuales y Nuevas Tecnologías de la misma Universidad, director del Grupo de Investigación Didáctica, ha realizado diferentes publicaciones sobre distintos aspectos de la Tecnología Educativa.

E-mail: cabero@us.es

\section{DIRECCIÓN DE LOS AUTORES}

Facultad de Ciencias de la Educación

Universidad de Sevilla

Cl. Pirotecnia, s/n

Sevilla (España)

Fecha de recepción del artículo: 25/12/2015

Fecha de aceptación del artículo: 01/04/2016

\section{Como citar este artículo:}

García Marcos, C. J., y Cabero Almenara, J. (2016). Evolución y estado actual del e-learning en la Formación Profesional española. RIED. Revista Iberoamericana de Educación a Distancia, 19(2), pp. 167-191. doi: http://dx.doi.org/10.5944 ried.19.2.15800 


\section{ANEXO}

\section{Cuestionario de la "Situación actual de la FP a distancia"}

Este cuestionario de 29 preguntas nos va a permitir recoger información acerca de cuál ha sido la evolución de las enseñanzas de FP a distancia en todas las Comunidades Autónomas desde sus inicios y en qué situación se encuentran dichas enseñanzas actualmente.

Los datos suministrados se utilizarán como parte de un estudio cuya publicación informaremos vía correo electrónico. Los datos personales únicamente se utilizarán como medio de contacto para nuestra investigación y en ningún caso se harán públicos.

El tiempo estimado para rellenarlo es de 10 minutos.

iMuchas gracias por su colaboración!

\section{Datos institucionales}

1. Nombre de la institución:

2. Comunidad Autónoma a la que pertenece:

\section{Datos personales (en ningún caso se harán públicos)}

3. Nombre:

4. Correo electrónico:

5. Cargo:

\section{Datos sobre la organización institucional de la FP a distancia}

6. ¿En qué curso se iniciaron las enseñanzas de FP a distancia en su Comunidad Autónoma? Ejemplo: Curso 2009/2010

7. ¿Existe algún tipo de cargo específico para la Coordinación de las enseñanzas de FP a distancia?

() Sí.

() No. Pase a la pregunta 9.

8. ¿Cuál es el modelo de organización utilizado para la Coordinación específica de la FP a distancia?

Ejemplos: existe un Coordinador de FP a distancia para toda la Comunidad Autónoma, existe un Coordinador de FP a distancia por centro y, además, un 
Coordinador superior de FP a distancia que organiza a estos Coordinadores de centro.

9. Para toda la Comunidad Autónoma, las enseñanzas de FP a distancia se imparten:

() De manera centralizada, desde un único centro educativo. Pase a la pregunta 11.

( ) De manera descentralizada, con un único centro educativo para cada especialidad (centros diferentes por especialidad).

( ) De manera descentralizada, con varios centros educativos para cada especialidad.

() Otros (indicar)

\section{Datos sobre la plataforma educativa (LMS)}

10. Respecto a la plataforma (LMS) que se utiliza para las enseñanzas de FP a distancia...

() Es única para toda la Comunidad Autónoma y de uso obligado para todos los centros.

() Hay disponible una para toda la Comunidad Autónoma, pero cada centro educativo tiene libertad para utilizar la suya propia.

11. ¿Desde qué curso se encuentra implantado el LMS que se utilizará durante el curso 2015/2016? Ejemplo: Curso 2012/2013

12. Anteriormente al LMS indicado en la pregunta anterior, cutilizaron algún otro?

() Sí.

() No. Pase a la pregunta 14.

13. ¿Qué otros LMS se han utilizado? Indique el nombre y ponga entre paréntesis el primer curso en qué se implantó. Ejemplo: Blackboard (2009/2010), Edmodo (2012/2013)

14. ¿Existe algún tipo de cargo específico para la administración del LMS?

() Sí, existe una persona encargada específicamente de la administración del LMS.

( ) Sí, existe un equipo de personas encargadas específicamente de la administración del LMS.

() No. Aunque existe una persona, también realiza otras tareas no relacionadas con la administración del LMS.

() No. Aunque existe un equipo de personas, también realiza otras tareas no relacionadas con la administración del LMS.

\section{Datos sobre los materiales didácticos}

15. ¿Existen materiales didácticos creados específicamente para las enseñanzas de FP a distancia? 

() Sí.
() No. Pase a la pregunta 22.

16. Los materiales didácticos han sido:

() Creados por un único centro, de manera centralizada.

() Creados por diversos centros, de manera descentralizada.

() Creados por terceras empresas.

() Otros (Indicar)

17. 17. ¿Los materiales didácticos están disponibles para el público en general?

() Sí.

() No.

18. ¿Los materiales didácticos poseen algún tipo de licencia libre? Ejemplos: Creative Commons, GNU/FDL.
() Sí.

() No.

19. ¿En qué formato se encuentran los materiales didácticos? Ejemplos: pdf, html.

20. ¿Existen mecanismos de actualización de los materiales didácticos?

() No.

() Sí. La actualización se realiza por un centro, de manera centralizada.

()Sí.Laactualización se realiza por diversos centros, demanera descentralizada.

() Sí. La actualización se realiza por terceras empresas.

() Otros (Indicar)

21. ¿Qué herramienta se utiliza para crear y/o actualizar los materiales didácticos? Ejemplos: eXeLearning, CourseLab, Xerte.

\section{Datos sobre el profesorado}

22. El profesorado que imparte enseñanzas de FP a distancia:

() Es un equipo creado y formado específicamente para este tipo de enseñanzas. Pase a la pregunta 24.

( ) No es específico para tal fin, pero debe impartir únicamente módulos en modalidad a distancia.

() No es específico para tal fin y puede tener módulos en modalidad presencial dentro su horario.

() Otros (Indicar)

23. La formación recibida por el profesorado que imparte enseñanzas de FP a distancia:

( ) Consiste en un curso de formación específico con una duración superior a las 100 horas.

( ) Consiste en un curso de formación específico con una duración entre 50 y 100 horas. 
() Consiste en un curso de formación específico con una duración inferior a 50 horas.

() El profesorado no recibe formación específica para la impartición a distancia. Otros (Indicar)

24. ¿Cuál es el número máximo de estudiantes en un grupo? NOTA: Por grupo entendemos la cantidad de estudiantes de un módulo asignados a un docente

25. ¿Cuántas horas lectivas por módulo tiene el profesorado que imparte FP a distancia?

( ) Las horas por módulo son las mismas que en la modalidad presencial, independientemente del número de alumnos en el grupo.

() Las horas por módulo duplican a las de la modalidad presencial.

() Otros (Indicar)

26. ¿Qué elementos de la plataforma (LMS) puede gestionar el profesor? Cuando hablamos de gestionar, nos referimos a dar de alta, modificar y eliminar.

() Plataforma, cursos y usuarios.

() Cursos y usuarios.

() Cursos.

() Usuarios.

() Ninguno.

() Otros (Indicar)

\section{Otras consideraciones}

27. ¿De qué otros recursos se dispone para las enseñanzas de FP a distancia? (puede marcarse varias casillas)

() Despachos para la atención individual del alumno.

() Salas para la atención grupal de los alumnos.

() Correo electrónico para alumnos y profesores.

() Software para alumnos y profesores (p.e.: sistema operativo, aplicaciones,...)

() Herramientas para el alumno (p.e.: ordenador, cuchillos de cocina, peines,...)

() Aplicaciones de gestión para el profesor (p.e.: seguimiento de alumnos).

() Otros (Indicar)

28. ¿Qué recursos se ponen a disposición del alumno para que pueda tenerlos en casa?

(puede marcarse varias casillas)

() Software (p.e.: sistema operativo, aplicaciones,...)

() Herramientas (p.e.: ordenador, cuchillos de cocina, peines,...)

() Otros (Indicar)

29. Por favor, si desea añadir cualquier comentario o información que considere relevante, hágalo a continuación. Gracias. 